\title{
The comparison of high and standard definition computed tomography techniques regarding coronary artery imaging
}

\author{
Aktas Aykut ${ }^{1}$, Degirmenci Bumin ${ }^{1}$, Yilmaz Omer $^{1}$, Kayan Mustafa $^{1}$, Cetin Meltem ${ }^{1}$, Celik Orhan ${ }^{1}$, \\ Unlu Nisa ${ }^{1}$, Orhan Hikmet ${ }^{2}$, Demirtas Hakan ${ }^{1}$, Koroglu Mert ${ }^{3}$
}

1. Suleyman Demirel University, Radiology Dept.

2. Suleyman Demirel University, Biostatistics Dept.

3. Near East University, Radiology Dept.

\begin{abstract} phy as to radiation dose, image quality and accuracy. independent samples t-test and Mann-Whitney $\mathrm{U}$ test. significant difference between image quality. measurement accuracy for imaging coronary arteries.

Key words: Coronary Arteries, Computerised Tomography, Angiography

DOI: http://dx.doi.org/10.4314/ahs.v15i3.29 $d x$.doi.org/10.4314/abs.v15i3.29

\section{Introduction}

In coronary artery disease follow-up, coronary CT angiography (CCTA) is a non-invasive method which becomes very important and useful in the assessment of coronary arteries ${ }^{1}$.

CCTA is highly accurate but image quality can be affected by several factors in some patients. As an example, highly calcified vessels, high body mass index, and higher heart rates causing excessive image noise, partial volume and beam hardening artifacts, and limited temporal resolution decrease diagnostic accuracy of CCTA $^{2}$.

Recently developed high definition CT(HDCT) allow-
\end{abstract}

Objective: The aim was to compare coronary high-definition CT (HDCT) with standard-definition CT (SDCT) angiogra-

Material and methods: 28 patients with history of coronary artery disease scanned by HDCT (Discovery CT750 HD) and SDCT (Somatom Definition AS). The scan modes were both axial prospective ECG-triggered. The vessel diameters and vessel attenuation values of totally 280 measurements from 140 coronary arteries were analyzed by two experienced radiologists. All data was analyzed by intraclass correlation test. Image quality graded by motion and stair step artifacts (grade 1, poor, to grade 4, excellent), accuracy of vessel inner and outer diameters were compared between the two CT units using the

Results: The intraclass correlation coefficient (ICC) of measured vessel attenuation values in SDCT between the two radiologists was exceedingly good. The ICC was higher in HDCT. The radiation dose of HDCT was higher than that of SDCT. The mean tube current was $180(\mathrm{~mA})$ in HDCT and $147(\mathrm{~mA})$ in SDCT with the same tube voltage $(\mathrm{kVp})$. There was no

Conclusion: HDCT has a higher radiation dose but has much more atenuation and the spatial resolution which improve

Cite as: Aykut A, Bumin D, Omer Y, Mustafa K, Meltem C, Orban C, Nisa U, Hikmet O, Hakan D, Mert K. The comparison of high and standard definition computed tomography techniques regarding coronary artery imaging. Afri Health Sci. 2015;15(3):925-30. doi: bttp://

\section{Corresponding author: \\ Aktas Aykut \\ Suleyman Demirel University, \\ Radiology Dept. \\ Email: aykutraktas@gmail.com}

es high spatial resolution imaging that improves Data Acquisition (DA). X-ray tube with the deflecting focal spot, 2496 sampling per rotation, and a new gemstone detector improved spatial and contrast resolution ${ }^{3}$. Standard definition computed tomography (SDCT) is known to be limited by spatial and contrast resolution in CCTA ${ }^{4,5}$.

The purpose of this clinical study was to compare HDCT and conventional 64-row SDCT for the performance of imaging coronary arteries according to image quality, diagnostic performance and radiation exposure.

\section{Materials and methods}

Patients with coronary heart disease history with stable heart rates of $60-80$ beats per minute were selected in different university hospitals by using contrast material iohexol(Omnipaque 350, GE Healthcare, Amersham, $\mathrm{UK})$, prospective ECG-triggered axial scans were performed in the same cardiac phase for both HDCT(120 $\mathrm{kV}, 180 \mathrm{~mA}$ ) (Discovery HD 750, GE Healthcare, Waukesha, WI) and SDCT $(120 \mathrm{kV}, 147 \mathrm{~mA})$ (Somatom Definition AS, Erlangen, Germany) with slice thick- 
ness of $0.675 \mathrm{~mm}$ and $0.6 \mathrm{~mm}$ respectively. Patients with unstable and rapid heart rate with motion artefacts and highly calcified coronary arteries were exluded from the study.

All images were analyzed by two radiologists(ARA. and BD.) with special training in cardiovascular imaging 3 and 5 years of experience, respectively. They were blinded with the CT protocols on the same viewing programme (Enlil PACS Viewer, Eroglu Yazılım, Eskisehir, Turkey). The observers measured inner and outer vessel diameters of the visible maximum diameter of vascular lumen of ascending aorta(AA), left main coronary artery (LM), left anterior descending coronary artery (LAD), left circumflex artery $(\mathrm{Cx})$, and right coronary artery (RCA) using electronic calipers and attenuation values by using a region of interest (ROI) technique inside the non-stented or calcified lumen of arteries on the plane perpendicular to the long-axis of the vessel (Figure 1).

The luminal diameters obtained at the $1 \mathrm{~cm}$ proximal
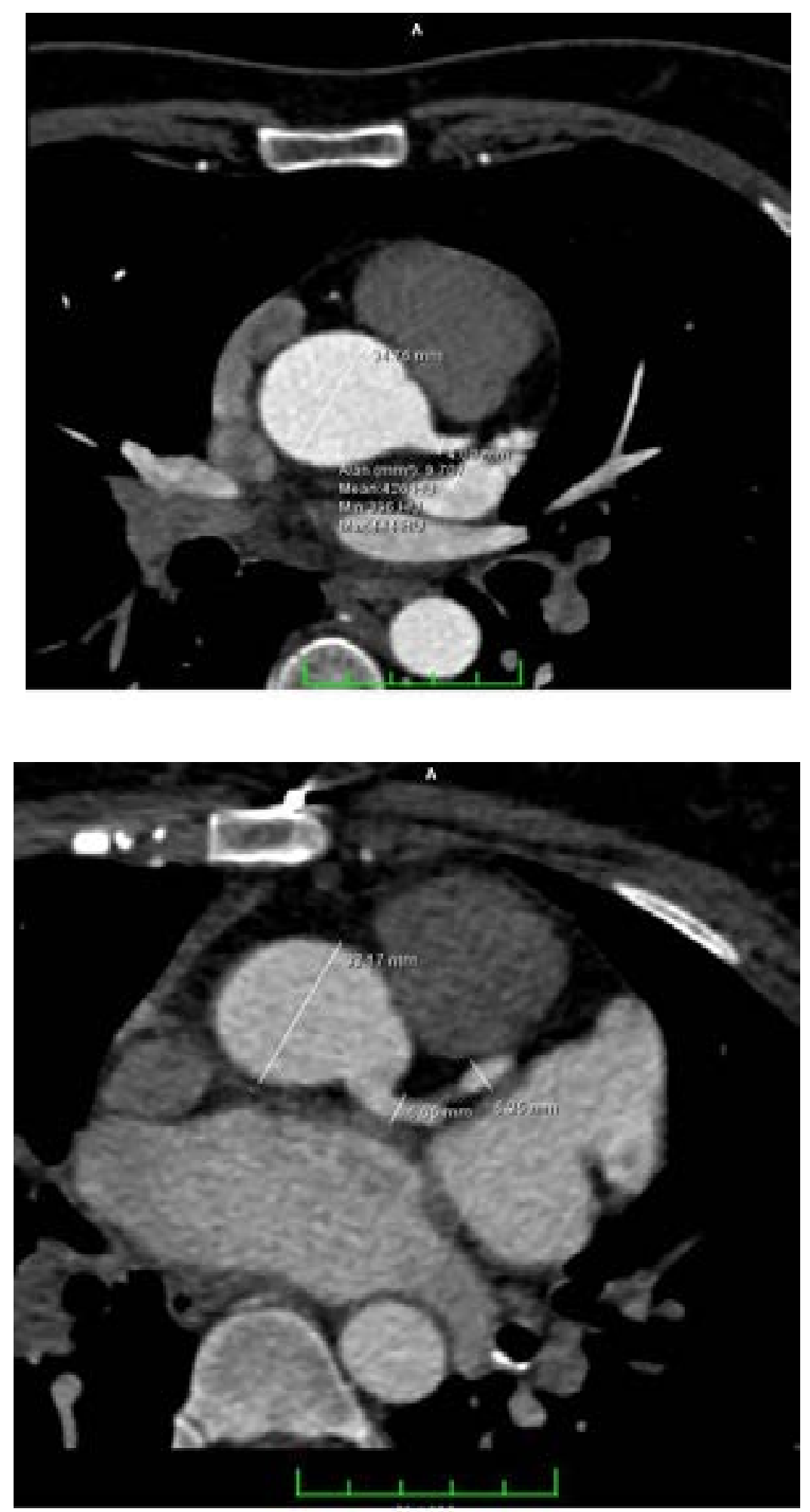
portions of each coronary vessel and the mean luminal diameters were also calculated. Image quality was graded and attributed in a 4-step scale (grade 4, excellent quality, vessels showing a continuous course, without stair-step artifacts; grade 3, good quality, presence of discrete blurring of vessel margin, minor motion artifacts; grade 2, moderate quality, noticeably blurred vessels or plaque margins, distinctly broader motion artifacts; and grade 1, poor quality, inadequate delineation between the vessel and surrounding tissue). Doselength product (DLP) was displayed on the dose report on the CT scans, and the effective dose $(\mathrm{E}=\mathrm{k} \times \mathrm{DLP}$, $\mathrm{k}=0.014 \mathrm{mSv}$ mGy-1cm-1 for the chest) was calculated. All the data was analyzed with the SPSS 11.0 software (Chicago, IL). Intraclass correlation coefficient (ICC) was used to compare the correlation between effects of the scanner within the radiologists' measurements of vascular diameters and attenuation values. Radiation dose in HDCT or SDCT were compared by independent samples t-test and Mann-Whitney U test. A 2-tailed $P$ value less than 0.05 was considered statistically significant.

\section{Results}

Totally 280 measurements from 140 coronary arteries of $18(64 \%)$ male and $10(36 \%)$ female patients with a median age of 51(42-76) were analyzed. The intraclass correlation coefficient (ICC) of measured vessel attenuation values in SDCT between the two radiologists was very good.Measurements of $\mathrm{Cx}, \mathrm{LAD}$ and LM artery were higher in HDCT but Aorta and RCA diameters were higher in SDCT (Table1).

The radiation dose of high definition CT angiography

Table 1: Independent samples t test

\begin{tabular}{|c|c|c|c|c|}
\hline \multirow[b]{2}{*}{ Variables } & \multicolumn{2}{|l|}{ CT type } & \multirow[b]{2}{*}{ Test } & \multirow[b]{2}{*}{$\mathrm{P}$} \\
\hline & $\operatorname{HD}(n=11)$ & $\mathrm{SD}(\mathrm{n}=17)$ & & \\
\hline age & $51,1 \pm 16,7$ & $50,9 \pm 13,5$ & 0,036 & 0,971 \\
\hline dose dlp & $1028(887-1350)$ & $461(289-676)$ & $-4,399$ & $<0,001$ \\
\hline dose $\mathrm{mSv} 1$ & $14,39(12,42-18,9)$ & $6,45(4,05-9,46)$ & $-4,399$ & $<0,001$ \\
\hline iq1 & $3(1-4)$ & $3(1-3)$ & $-0,857$ & 0,392 \\
\hline Tube curr 1 & $180(180-200)$ & $147(67-183)$ & $-4,044$ & $<0,001$ \\
\hline Aorta Vod 1 & $37,65 \pm 6,87$ & $35,36 \pm 5,71$ & 0,956 & 0,348 \\
\hline Aorta Vid1 & $32,8(25,5-46,1)$ & $32,2(24,8-47,8)$ & $-0,565$ & 0,572 \\
\hline Aorta HU1 & $373,82 \pm 74,36$ & $353,18 \pm 94,02$ & 0,613 & 0,545 \\
\hline LM Vod1 & $5,86 \pm 1,17$ & $5,48 \pm 0,94$ & 0,95 & 0,351 \\
\hline LM Vid1 & $4,19 \pm 0,96$ & $3,81 \pm 1,09$ & 0,943 & 0,354 \\
\hline LM HU1 & $364,09 \pm 66,79$ & $325,94 \pm 104,37$ & 1,068 & 0,296 \\
\hline LAD Vod 1 & $4,75 \pm 1,29$ & $4,58 \pm 0,73$ & 0,452 & 0,655 \\
\hline LAD Vid1 & $3,3(2,4-6,2)$ & $3,6(1,7-4,4)$ & $-0,141$ & 0,888 \\
\hline LAD HU1 & $334(258-447)$ & $316(126-952)$ & $-0,753$ & 0,452 \\
\hline Cx Vod1 & $4,33 \pm 0,97$ & $3,94 \pm 0,85$ & 1,126 & 0,270 \\
\hline Cx Vid1 & $3,10 \pm 0,87$ & $2,65 \pm 0,91$ & 1,31 & 0,202 \\
\hline Cx HU1 & $339,64 \pm 77,86$ & $315,00 \pm 109,33$ & 0,642 & 0,527 \\
\hline RCA Vod1 & $4,79 \pm 0,97$ & $4,07 \pm 0,71$ & 2,273 & 0,032 \\
\hline RCA Vid1 & $3,53 \pm 0,84$ & $2,74 \pm 0,83$ & 2,458 & 0,021 \\
\hline RCA HU1 & $318(285-432)$ & $316(178-514)$ & $-0,682$ & 0,495 \\
\hline iq2 & $2(1-4)$ & $3(1-4)$ & $-0,539$ & 0,590 \\
\hline Aorta Vod2 & $40,3(31-47)$ & $33(26,3-53)$ & $-1,863$ & 0,062 \\
\hline Aorta Vid2 & $35,87 \pm 5,98$ & $31,06 \pm 3,71$ & 2,391 & 0,030 \\
\hline Aorta HU2 & $326,09 \pm 97,88$ & $367,18 \pm 81,96$ & $-1,201$ & 0,241 \\
\hline LM Vod2 & $5,95 \pm 1,01$ & $4,84 \pm 1,27$ & 2,445 & 0,022 \\
\hline LM Vid2 & $4,44 \pm 1,01$ & $3,83 \pm 1,07$ & 1,521 & 0,140 \\
\hline LM HU2 & $299(148-475)$ & $325(210-550)$ & $-0,612$ & 0,541 \\
\hline LAD Vod 2 & $4,82 \pm 1,00$ & $3,74 \pm 0,67$ & 3,446 & 0,002 \\
\hline LAD Vid2 & $3,65 \pm 0,77$ & $2,72 \pm 0,51$ & 3,815 & 0,001 \\
\hline LAD HU2 & 334 (147-900) & $334(220-925)$ & $-0,26$ & 0,795 \\
\hline Cx Vod2 & $4,21 \pm 0,94$ & $3,25 \pm 0,59$ & 3,36 & 0,002 \\
\hline $\mathrm{Cx} \operatorname{Vid} 2$ & $3,29 \pm 0,76$ & $2,48 \pm 0,50$ & 3,45 & 0,002 \\
\hline Cx HU2 & $302,36 \pm 102,50$ & $334,38 \pm 87,27$ & $-0,873$ & 0,391 \\
\hline RCA Vod2 & $4,80 \pm 1,57$ & $3,65 \pm 0,90$ & 2,194 & 0,045 \\
\hline RCA Vid2 & $3,51 \pm 1,14$ & $2,75 \pm 0,81$ & 2,06 & 0,050 \\
\hline RCA HU2 & $294,27 \pm 77,56$ & $323,88 \pm 88,75$ & $-0,904$ & 0,374 \\
\hline
\end{tabular}

iq: image quality, Vod: Vessel outer diameter, Vid: Vessel inner diameter, HU: attenuation value 1: first radiologist measurement 2 : second radioligist measurement 
(HDCTA) $(14,3 \mathrm{mSv})$ was higher than that of SDCTA $(6,5 \mathrm{mSv})$. The mean tube current was $180(\mathrm{~mA})$ in
HDCT and $147(\mathrm{~mA})$ in SDCT when the same voltage $(\mathrm{kVp})$ were used in both methods (Table 2$)$.

There was no important difference between image

Table 2: Intraclass Correlation Coefficient (ICC)

\begin{tabular}{lll}
\hline & CT type & \\
\cline { 2 - 3 } Variables & HD $(\mathrm{n}=11)$ & SD $(\mathrm{n}=17)$ \\
\hline Aorta Vod & 0,337 & 0,777 \\
Aorta Vid & 0,152 & 0,677 \\
Aorta HU & 0,288 & 0,908 \\
LM Vod & 0,665 & 0,621 \\
LM Vid & 0,498 & 0,571 \\
LM HU & 0,080 & 0,969 \\
LAD Vod & 0,741 & $-0,334$ \\
LAD Vid & 0,434 & 0,322 \\
LAD HU & $-0,140$ & 0,960 \\
Cx Vod & 0,832 & 0,466 \\
Cx Vid & 0,674 & 0,582 \\
Cx HU & 0,327 & 0,887 \\
RCA Vod & 0,684 & 0,716 \\
RCA Vid & 0,592 & 0,831 \\
RCA HU & 0,313 & 0,916 \\
\hline
\end{tabular}

iq: image quality, Vod: Vessel outer diameter, Vid: Vessel inner diameter, HU: attenuation value 1: first radiologist measurement 2: second radioligist measurement

quality assesments of radiologists and attenuation val- beats per minute (bpm) when using the minimal X-ray ues of HDCT and SDCT in stable heart rate up to 75 exposure time (table 3).

\section{Discussion}

Table 3: The measurements of two radiologists from both type of CT scanners

\begin{tabular}{|l|l|l|l|l|}
\hline & HDCT & SDCT & test & $p$ \\
\hline Tube curr1 & $180(180-200)$ & $147(67-183)$ & $-4,044$ & $<0,001$ \\
\hline dose mSv1 & $14,39(12,42-18,9)$ & $\begin{array}{l}6,45 \quad(4,05- \\
9,46)\end{array}$ & $-4,399$ & $<0,001$ \\
\hline iq1 & $3(1-4)$ & $3(1-3)$ & $-0,857$ & 0,392 \\
\hline iq2 & $2(1-4)$ & $3(1-4)$ & $-0,539$ & 0,59 \\
\hline Aorta HU1 & $373,82 \pm 74,36$ & $353,18 \pm 94,02$ & 0,613 & 0,545 \\
\hline Aorta HU2 & $326,09 \pm 97,88$ & $367,18 \pm 81,96$ & $-1,201$ & 0,241 \\
\hline Cx HU1 & $339,64 \pm 77,86$ & $315,00 \pm 109,33$ & 0,642 & 0,527 \\
\hline Cx HU2 & $302,36 \pm 102,50$ & $334,38 \pm 87,27$ & $-0,873$ & 0,391 \\
\hline LAD HU1 & $334(258-447)$ & $316(126-952)$ & $-0,753$ & 0,452 \\
\hline LAD HU2 & $334(147-900)$ & $334(220-925)$ & $-0,26$ & 0,795 \\
\hline LM HU1 & $364,09 \pm 66,79$ & $325,94 \pm 104,37$ & 1,068 & 0,296 \\
\hline LM HU2 & $299(148-475)$ & $325(210-550)$ & $-0,612$ & 0,541 \\
\hline RCA HU1 & $318(285-432)$ & $316(178-514)$ & $-0,682$ & 0,495 \\
\hline RCA HU2 & $294,27 \pm 77,56$ & $323,88 \pm 88,75$ & $-0,904$ & 0,374 \\
& & & & \\
& & & & \\
\hline
\end{tabular}

iq: image quality, Vod: Vessel outer diameter, Vid: Vessel inner diameter, HU: attenuation value 1: first radiologist measurement 2 : second radioligist measurement 
Coronary artery disease (CAD) is one of the major causes of death and recently the 64-slice multi-detector row computed tomography (MDCT) permits successful evaluation of coronary artery. The diagnostic performance of MDCT for coronary artery evaluation have shown high accuracy for the detection of obstructive coronary artery stenosis in general patient populations ${ }^{6,7}$.

The reported sensitivity was between $86-99 \%$ and specificity $93-97 \%$. The negative predictive value was 95-99\% that suggests coronary CT angiography (CTA) has the potential to rule out the presence of coronary stenosis ${ }^{8,9}$.

But MDCT has a limited temporal and spatial resolutions compared with catheter angiography that reduced diagnostic performance of coronary CTA. The accuracy for detection of coronary stenosis is lower in the presence of severe calcification ${ }^{10,11}$.

HDCT scanner with high sampling rate data acquisition system, X-ray tube with the deflecting focal spot and a gemstone detector, improved the in-plane spatial resolution to $0.23 \mathrm{~mm}$ and a contrast resolution to $3 \mathrm{~mm}$. The gemstone detector is made from a complex rare earth particle which has a garnet crystal structure. The Gemstone detector has the direct effect of decreasing in-plane spatial resolution ${ }^{12}$.

While spatial and temporal resolution increase, there is an increase in radiation dose ${ }^{13}$. The exposure to radiation has to be as low as reasonably achievable (ALARA). In our study, the effective dose is higher than that for diagnostic coronary angiography reported that is $2.1 / 2.5 \mathrm{mSv}$ (male/female) ${ }^{14}$. The radiation dose varies with the square of the kilo voltage. For reduction of radiation dose, we should have used lower tube voltage such as $80 \mathrm{kV}$ or $100 \mathrm{kV}$. Furthermore, the decreased tube voltage leads to increased opacification of vascular structures during contrast-enhanced CTA owing to an increase in the photoelectric effect and a decrease in Compton scattering ${ }^{15}$.

\section{Limitation}

The first limitation of our study, $120 \mathrm{kVp}$ was mainly used although no tube voltage value had been widely accepted as standard for coronary artery imaging. We have found that HDCT has also more tube current so that the effective dose was higher. Second, since we focus on spatial resolution on coronary artery imaging, the impact of temporal resolution was not mentioned, and no specific heart rate had been used. Third, we studied different patients from different hospitals so that there was no physical unity. The patients had wider AA and RCA in the SDCT compared to HDCT. Last and the most important limitation was the hardware problem so we could not be able to use a novel ASIR method to reconstruct CT images.

HDCT has a higher radiation dose than SDCT but has much more attenuation, and the spatial resolution was estimated to be $0.71 \mathrm{~mm}$ on SDCT and $0.50 \mathrm{~mm}$ on HDCT which improve measurement accuracy for imaging coronary arteries.

ASIR techniques are theoretically more accurate in the modeling of physical noise and tissue geometries. Prior in vitro studies have shown improved image quality for enhanced image resolution as well as lower image noise by use of these Bayesian iterative algorithms ${ }^{16}$.

\section{Conclusion}

There are few clinical studies comparing HDCT with SDCT. Because it is hard to find both devices in the same center and the radiation dose is much higher when applying both scans to one patient. We compared the radiologist image quality assessment and correlations of vascular diameter measurements. HDCT offers a little improved measurement accuracy for imaging coronary arteries compared to conventional SDCT. The diagnostic performance of HDCT for coronary artery imaging compared to SDCT is better, but ASIR is mandatory for a lower radiation dose.

\section{References}

1. Schuijf JD, Pundziute G, Jukema JW, Lamb HJ, Tuinenburg JC, van der Hoeven BL, et al. Evaluation of patients with previous coronary stent implantation with 64-section CT. Radiology.2007;245:416- PubMed ;423. 2. G.L. Raff, M.J. Gallagher, W.W. O'Neill, J. Goldstein Diagnostic accuracy of noninvasive coronary angiography using 64-slice spiral computed tomography. J Am Coll Cardiol 2005;46: 552- PubMed ;557

3. Vartuli JS, Lyons RJ, Vess CJ et al (2008) GE Healthcare's New Computed Tomography Scintillator-Gemstone. Symposium on Radiation Measurement and Applications, June 2-5, Berkeley, California

4. Wykrzykowska JJ, Arbab-Zadeh A, Godoy G, Miller JM, Lin S, Vavere A, et al. Assessment of in-stent restenosis using 64-MDCT: analysis of the CORE-64 Multicenter International Trial. AJR Am J Roentgenol. 2010;194:85- PubMed ;92.

5. Wen Jie Yang, Ke Min Chen, Li Fang Pang, Ying Guo, 
Jian Ying Li, Huang Zhang, Zi Lai Pan. High-Definition Computed Tomography for Coronary Artery Stent Imaging: a Phantom Study. Korean J Radiol. 2012 ; 13: 20-26.

6. M.J. Budoff, D. Dowe, J.G. Jollis, M. Gitter, J. Sutherland, E. Halamert, M. Scherer, R. Bellinger, A. Martin, R. Benton, A. Delago, J.K. Min Diagnostic performance of 64-multidetector row coronary computed tomographic angiography for evaluation of coronary artery stenosis in individuals without known coronary artery disease: results from the prospective multicenter ACCURACY (Assessment by Coronary Computed Tomographic Angiography of Individuals Undergoing Invasive Coronary Angiography) trial. J Am Coll Cardiol 2008;52:1724- PubMed ;1732

7. M. Hamon, G.G. Biondi-Zoccai, P. Malagutti, P. Agostoni, R. Morello, M. Valgimigli, M. Hamon Diagnostic performance of multislice spiral computed tomography of coronary arteries as compared with conventional invasive coronary angiography: a meta-analysis. J Am Coll Cardiol 2006;48:1896- PubMed ;1910

8. Ropers D, Rixe J, Anders K, et al. Usefulness of multidetector row computed tomography with $64-\times$ 0.6-mm collimation and 330-ms rotation for the noninvasive detection of significant coronary artery stenoses. Am J Cardiol 2006 ;97 : 343-348

9.Fine JJ, Hopkins CB, Ruff N, Newton FC. Comparison of accuracy of 64-slice cardiovascular computed tomography with coronary angiography in patients with suspected coronary artery disease. Am J Cardiol 2006; $97: 173-174$

10. Hoffmann U, Moselewski F, Cury RC, et al. Pre- dictive value of 16-slice multidetector spiral computed tomography to detect significant obstructive coronary artery disease in patients at high risk for coronary disease: patient versus segment-based analysis. Circulation 2004;110:2638\& PubMed nbsp;-2643

11. Cordeiro MA, Miller JM, Schmidt A, et al. Noninvasive half millimetre 32 detector row computed tomography angiography accurately excludes significant stenoses in patients with advanced coronary artery disease and high calcium scores. Heart 2006;92:589- PubMed ;597

12. Yang, W.Chen, K.Pang, L.Pan, High-Definition Computed Tomography for Coronary Artery Stent Imaging: a phantom study. Korean J Radiol. 2012; 13: 20 PubMed ;26.

13. Hausleiter J, Meyer T, Hadamitzky M, et al. Radiation dose estimates from cardiac multislice computed tomography in daily practice: impact of different scanning protocols on effective dose estimates. Circulation 2006;113:1305- PubMed ;1310

14. Coles DR, Smail MA, Negus IS, et al. Comparison of radiation doses from multislice computed tomography coronary angiography and conventional diagnostic angiography. J Am Coll Cardiol 2006; 47:1840 -1845

15. Ertl-Wagner BB, Hoffmann RT, Bruning R, et al. Multi-detector row CT angiography of the brain at various kilo voltage settings. Radiology 2004; 231: 528-535 16. J.B. Thibault, K.D. Sauer, C.A. Bouman, J. Hsieh A three-dimensional statistical approach to improved image quality for multislice helical CT. Med Phys 2007; 34: 4526- PubMed ;4544 